\title{
Perspective
}

PERSPECTIVE Actualité en histoire de l'art

2| 2007

La Grande-Bretagne/Période moderne

\section{La presse artistique au Royaume-Uni}

\section{Martin Patrick}

\section{OpenEdition}

\section{Journals}

Édition électronique

URL : http://journals.openedition.org/perspective/3822

DOI : 10.4000/perspective.3822

ISSN : 2269-7721

Éditeur

Institut national d'histoire de l'art

Édition imprimée

Date de publication : 30 juin 2007

Pagination : 318-321

ISSN : 1777-7852

\section{Référence électronique}

Martin Patrick, «La presse artistique au Royaume-Uni », Perspective [En ligne], 2 | 2007, mis en ligne le 31 mars 2018, consulté le 01 octobre 2020. URL : http://journals.openedition.org/perspective/3822 ; DOI : https://doi.org/10.4000/perspective.3822 


\title{
La presse artistique au Royaume- Uni
}

\author{
Martin Patrick
}

1 Les critiques d'art britanniques n'ont jamais eu leur pareil pour mêler, dans leurs discussions et leurs débats, esprit caustique et vaste culture historique. Aujourd'hui cependant, la plupart des revues d'art, en Grande-Bretagne comme partout ailleurs, ne sont qu'une compilation de textes descriptifs qu'on lit aussi vite qu'on les oublie et qui disparaissent derrière une masse gigantesque de publicité. Il faut dire qu'il revient extrêmement cher de publier à une fréquence mensuelle ou trimestrielle la centaine de pages de papier glacé et de reproductions couleur qui composent un magazine. Et le prix à payer n'est pas seulement financier: la presse artistique à fort tirage tend à perdre en substance, car elle fait l'impasse sur la réflexion polémique et se consacre plutôt à l'information et à la publicité.

2 Ce n'est pourtant pas le cas d'Art Monthly (www.artmonthly.co.uk), le plus ancien des magazines d'art britanniques. Dans un communiqué publié à l'occasion du trentième anniversaire de la revue, Patricia Bickers, la rédactrice en chef, affirme qu'elle " persiste à publier Art Monthly en noir et blanc, parce que l'essentiel demeure la critique, les idées, le débat et l'analyse. Même lorsqu'elle est bien faite, la critique d'art ne doit pas se substituer au contact avec les œuvres, mais au contraire stimuler le lecteur $»^{1}$. Cette déclaration semble être dans la droite ligne du projet éditorial initial d' Art Monthly. Le fondateur du magazine, Peter Townsend, était en effet un homme engagé et d'une très grande culture, qui avait vécu plusieurs années en Chine; il décrivait le périodique, à sa première parution, comme « une nouvelle revue de débat, pas chère, et qu'on pouvait jeter après l'avoir lue $»^{2}$.

3 N'étant pas une publication à but lucratif, Art Monthly dut sa subsistance à la générosité d'un couple de collectionneurs et galeristes, Jack et Nell Wendler (cette dernière a disparu en 2003), et à l'aide de l'Arts Council of Great Britain. Cela permit à la revue d'épargner à ses lecteurs le déluge d'encarts publicitaires ineptes dont sont remplis la plupart des magazines d'art à visée commerciale. Art Monthly put aussi suivre l'actualité de la scène artistique britannique de manière plus variée et plus indépendante (je dois 
d'ailleurs dire que j'ai écrit quelques textes pour eux et que j'ai été très satisfait de cette collaboration).

4 Art Monthly accorde plus de place au débat critique que n'importe lequel de ses concurrents sur le marché. Le magazine est connu pour être proche du courant minimaliste et conceptuel, les Wendler ayant en effet été les amis et mécènes de Marcel Broodthaers, entre autres artistes. La revue reste, à bien des égards, complètement à l'écart des modes, mais semble s'enorgueillir de cette image. Matthew Collings, critique d'art (et animateur de nombreuses émissions de télévision sur l'art contemporain), n'a d'ailleurs pas manqué de s'en moquer voilà quelques années : «Pendant longtemps, Art Monthly a voulu définir ce que devait être l'art. Puis soudainement, il n'y en eut plus que pour le Young British Art. Mais les articles sur la nature et le but de l'art sont restés des textes uniques en leur genre, tant ils sont lourds, voire assommants ${ }^{3}$.

5 Les rédacteurs en chef d'Art Monthly sont consciencieux au point de flirter parfois avec une inconscience salutaire, lorsque par exemple ils font figurer dans leur publication les échanges passionnés de leurs collaborateurs et d'autres critiques sur les politiques culturelles au Royaume-Uni. On trouve aussi l'inoxydable rubrique de Henry Lydiate sur la législation des biens culturels, sujet rarement abordé dans le reste de la presse artistique. En 2006, le trentième anniversaire de la revue coöncida avec le départ de son rédacteur en chef adjoint, Andrew Wilson (remplacé aujourd'hui par Ian Hunt), qui devint conservateur de la collection d'art con-temporain britannique à la Tate Gallery. La longévité d'Art Monthly constitue en elle-même un exploit, dans un domaine où l'éphémère semble être la règle.

6 L'histoire de Modern Painters (www.modernpainters.co.uk) est elle aussi intéressante et représentative de l'évolution de la presse artistique britannique. Il serait difficile, en feuilletant ce magazine aujourd'hui, d'y retrouver la moindre trace de l'esprit de son fondateur, Peter Fuller. Celui-ci n'était âgé que de quarante-quatre ans au moment de sa disparition en 1990, mais il avait déjà une réputation sulfureuse, acquise moins à cause de sa productivité et de son talent de critique que de son singulier cheminement, du marxisme et de la psychanalyse à un conservatisme esthétique parfois mêlé de rancœur. Fuller choisit le titre de son magazine, Modern Painters, en hommage au célèbre livre de John Ruskin, car il avait décidé de le consacrer à une vision plus traditionnelle de l'art et de la création plastique.

7 Vers la fin des années 1990, le magazine adopta une ligne éditoriale moins ouvertement conservatrice, avec une maquette plus attrayante et une équipe de collaborateurs éclectique, du philosophe Richard Wollheim (aujourd'hui disparu) au chanteur David Bowie - tous deux membres du comité de rédaction. Je peux témoigner du fait que les lecteurs les plus jeunes, en l'occurrence mes étudiants en arts plastiques, appréciaient beaucoup le magazine à l'époque, car il parlait de peinture sans se prendre pour autant au sérieux.

8 Très récemment, la rédaction de Modern Painters a quitté Londres pour Manhattan et abandonné sa formule trimestrielle pour devenir un mensuel. Elle a également supprimé de la revue la référence à Fuller qui était jusque là demeurée en couverture et s'est autoproclamée "magazine international d'art contemporain». Fuller s'était pourtant fait connaître comme le défenseur acharné de l'art «britannique » - pour autant qu'on puisse parler d'un art britannique. Une innovation des plus appréciables a été l'ajout de deux rubriques consacrées aux jeunes artistes. Au travers de tous ces 
changements, le magazine a cherché à se renouveler, après bientôt vingt années d'existence.

Comme Modern Painters, le magazine Art Review (www.artreviewdigital.com) a connu une évolution radicale en 2006. On peut à présent voir en couverture des artistes de stature internationale comme Annette Messager, Tim Hawkinson, Sam Durant, John Bock et même le réalisateur David Lynch. Comme Art Monthly, le magazine est à présent disponible en version électronique, téléchargeable en format pdf moyennant le paiement d'un abonnement. Lorsque je lis la presse artistique britannique, j'ai par moments la même impression que lorsque je regarde un téléfilm de la $\mathrm{BBC}$ - tous les acteurs me semblent familiers. Il y a finalement peu de critiques d'art sur le marché, et les mêmes noms reviennent, avec un nombre de permutations limité, au point que j'en oublie parfois quel magazine je suis en train de lire. Certains auteurs sont aujourd'hui partout, comme Brian Dillon, Martin Herbert, J. J. Charlesworth ou Julian Stallabrass.

10 À mon humble avis, tous ces changements ont été pour le mieux, même si les deux dernières revues que j'ai évoquées se ressemblent trop. On dirait qu'elles ont toutes deux pris pour modèle la nouvelle formule de Frieze (www.frieze.com). Il est presque aussi difficile de trouver un article de fond dans cette dernière que dans Artforum, revue new-yorkaise dont le dernier numéro, épais de plusieurs centimètres, ressemblait plus à un catalogue de vente par correspondance qu'à un magazine d'art. Frieze est d'ailleurs une véritable publication commerciale, surtout depuis qu'a été lancée, en octobre 2003, la Frieze Art Fair, foire d'art contemporain annuelle dont la dernière édition, sur cinq jours, a attiré des dizaines de milliers de visiteurs.

11 Frieze a été créé en 1991 par Amanda Sharp et Matthew Slotover, deux étudiants fraîchement diplômés d'Oxford. À l'origine, le magazine ressemblait presque à un fanzine, avec peu de pages, mais un graphisme extrêmement soigné, un ton désinvolte, informel, et des textes remarquablement impertinents. Y furent publiés les premiers articles de fond sur les Young British Artists (ou, comme on les appelle à présent, les YBAs). On trouvait souvent en couverture, à l'époque, des détails d'œuvres d'artistes comme Damien Hirst, Mark Wallinger ou Gavin Turk. Les rédacteurs en chef bénéficièrent rapidement $d u$ soutien d'un certain nombre d'acteurs de la scène artistique britannique et, dans les premières années d'existence du magazine, ils publièrent des textes d'auteurs comme Stuart Morgan (1948-2002), considéré comme un des critiques d'art majeurs des années 1980. Les derniers changements qu'a connus Frieze ont porté sur sa maquette et sa ligne éditoriale : nouvelle police de caractère, très petite et étroite, inclusion de projets d'artistes et nouvelles rubriques, dont celles $\mathrm{du}$ commissaire d'exposition américain Robert Storr et d'Adrian Searle, le critique d'art du Guardian.

12 Le problème, pour toutes les grandes revues d'art (celles qui sont distribuées à l'international et coûtent si cher à publier) est de savoir quelle place donner à la critique d'art alors que toutes, sans qu'on puisse aller jusqu'à parler de compromission, entretiennent d'étroites relations avec le marché. L'usage veut que l'on place la publicité dans les premières pages et la section «critiques et comptes rendus » en fin de magazine - dans l'une comme l'autre, on est sûr de retrouver les mêmes noms de galeries. Bien entendu, il y a de nos jours autant de publicité pour des marques comme Prada ou Comme des Garçons que pour la Lisson Gallery ou la Whitechapel Gallery. Même si Sharp et Slotover ne s'occupent plus vraiment du contenu éditorial de la revue 
mais plutôt de la partie commerciale, Frieze est l'exemple parfait de la manière dont le marché a fait tomber la critique d'art de son piédestal.

13 Contemporary ( www.contemporary-magazine.com), autre magazine londonien (autrefois appelé Contemporary Visual Arts), a été relancé en 2002, dans le but de toucher un public international. Il se définit comme " un magazine éclectique, abordant les arts plastiques, l'information, l'édition, les dernières tendances, l'architecture, le design, la mode, le cinéma, la musique, les nouvelles technologies, la photographie, la danse, le sport, et bien d'autres choses encore» (sic). La ribambelle de thèmes traités montre d'emblée que les revues d'art ne sont pas si différentes des magazines généralistes. D'où vient cette peur de ne parler que d'art? Elle est sans doute moins due à un désir d'interdisciplinarité qu'à un besoin de séduire un public le plus large possible, pour des raisons économiques évidentes. Toutefois, Contemporary a eu la bonne idée d'organiser chacun de ses numéros autour d'un sujet en particulier : ainsi, dernièrement, ont été abordés l'art numérique, les performances d'artistes, les relations entre art et architecture et l'art conceptuel. La plus prometteuse des nouvelles revues est sans aucun doute Afterall (www.afterall.org). L'équipe de rédaction - composée d'un commissaire d'exposition, Charles Esche, un peintre et critique d'art, Thomas Lawson, et un artiste, Mark Lewis travaille avec des collaborateurs d'horizons et de nationalités variés, parmi lesquels des critiques, des historiens, des conservateurs et des commissaires d'exposition. Plus volumineuse qu'une revue ordinaire, Afterall est le fruit du travail commun de deux écoles d'art, la Central Saint Martin's School of Art and Design et le California Institute of the Arts. Elle paraît à présent trois fois par an, grâce au soutien de la Andy Warhol Foundation, et devrait bientôt fusionner avec la revue anversoise Andere Sinema.

L'un des derniers numéros analysait magistralement la place de l'esthétique relationnelle dans la gestion des publics. L'étude, menée par Lane Relyea, critique d'art enseignant la théorie critique à Northwestern University (Chicago), mettait en parallèle la création d'une ambiance «lounge» dans les lieux d'exposition et l'utilisation par les multinationales d'espaces de détente similaires - une comparaison qui fait froid dans le dos. Le reste du numéro proposait un gros plan sur une demi-douzaine d'artistes travaillant en collectif ou sur des problématiques sociopolitiques, chacun d'entre eux (Andrea Bowers, Ibon Aranberri, le collectif Bernadette Corporation, Ivan Grubanov et Julie Mehretu) faisant l'objet de deux articles.

16 La démarche de la revue se comprend d'autant mieux si l'on considère les ouvrages de la collection Afterall Books, fondée sur un principe original : un critique de renommée internationale commente et analyse le travail d'un artiste à travers l'étude d'une de ses œuvres. Les premières œuvres à avoir été ainsi étudiées ont été The Man Who Flew into Space from his Apartment, de Ilya Kabakov (par Boris Groys), Untitled (couple) de Richard Prince (par Michael Newman) et In Search of the Miraculous, de Bas Jan Ader (par Jan Verwoert). Les éditeurs espèrent publier une centaine de titres dans cette collection.

Tout cela nous amène à un autre problème de taille, sur lequel nous terminerons cette réflexion: peut-on vraiment, à l'heure actuelle, parler de magazine d'art britannique? Et cela voudrait-il seulement dire quelque chose, dans un monde de l'art où la dispersion et le nomadisme semblent prévaloir? Les professionnels de l'art travaillent à l'échelle planétaire, pour des publications dont les comités de rédaction et les bureaux sont éparpillés dans plusieurs pays. Cette mondialisation de la critique d'art 
semble rendre inepte l'idée même qu'une revue puisse appartenir à un lieu donné et y ancrer son contenu.

Amanda Sharp, co-fondatrice de Frieze, a récemment déclaré dans une interview: "Depuis le début, il nous a semblé clair que Frieze ne serait pas un magazine d'art britannique, parce que nous avions compris que l'essence même de l'art contemporain était d'être international. Cela paraissait absurde de créer un magazine provincial $»^{4}$. La tendance actuelle à faire des magazines des entités à la fois hybrides - entre formule papier, version électronique et blogs - et internationales rend l'identité de la presse artistique britannique plus floue et plus insaisissable que jamais. Et c'est, me semble-til, une chose dont il faut se réjouir.

\section{NOTES}

1. Patricia Bickers, «Éditorial », dans Art Monthly, 300, octobre 2006, p. 19.

2. «Obituary : Peter Townsend ", dans The Times, 24 juillet 2006.

3. Matthew Collings, BLIMEY! From Bohemia to Britpop: The London Artworld from Francis Bacon to Damien Hirst, Cambridge, 1997, p. 47.

4. «London Special 3: Interview with FRIEZE co-founder Amanda Sharp », 18 octobre 2006, http://www.artfacts.net

\section{INDEX}

Index géographique : Grande-Bretagne

Keywords : magazines, art magazine, artistic press, art critic, art market, publicity, popularization, diffusion

Mots-clés : magazines, revues d'art, presse artistique, critique d'art, marché de l'art, publicité, vulgarisation, diffusion

Index chronologique : 1900, 2000

\section{AUTEUR}

\section{MARTIN PATRICK}

University of Chicago

fr 